\title{
DECISIONS
}

\section{Clicking hip in a postmenopausal woman}

\author{
Len Kelly MD MCISc, Rajiv Gandhi MD, Anukul Panu MD
}

\begin{abstract}
A 55-year-old postmenopausal woman experiences intermittent anterior clicking and locking of her hip while walking. The clicking is painful and comes on without warning, causing her to stop walking for several minutes. The patient has no history of trauma and no night pain. She enjoys golf, but now has to ride in a golf cart to complete the round. Initial examination shows a normal range of motion without pain. There is no evidence of femoral or inguinal hernia or localized tenderness consistent with a trochanteric bursitis. Radiographs of her hip appear normal.
\end{abstract}

\section{What causes of pain should be considered?}

The patient's history of intermittent symptoms indicates a mechanical origin of pain. That the patient had normal range of motion without pain on examination is a pertinent negative finding for osteoarthritis. Because there was no evidence of osteoarthritis or metastatic disease on the plain radiographs, nor evidence of hernia (femoral or inguinal), trochanteric bursitis or local inflammation or infection on examination, a labral tear of the cartilage of the acetabulum should be considered.

A labral tear occurs at the relatively avascular proximal portion of the cartilaginous labrum where it attaches to the articular cartilage of the hip joint. It typically presents as anterior mechanical hip pain.

The labrum acts as a seal of the synovial fluid and extends the depth of the joint by as much as $25 \%$. Pivoting in activities such as golf, hockey, soccer and ballet may cause tears. ${ }^{1,2}$ Two common types of tears are described: traumatic tears (young athletes) and degenerative tears (early osteoarthritis in older patients). ${ }^{1,3}$

Labral tears may be asymptomatic. In a study involving 70 asymptomatic individuals (mean age $26 \mathrm{yr}$ ), a surprising $27(39 \%)$ cases of labral tear seen on high-resolution magnetic resonance (MR) imaging were reported. ${ }^{4}$

\section{Does this patient require any additional assessment on examination?}

The clinical assessment for a labral tear involves evocative rotational end-point testing of a flexed hip. This test comprises stressing a $90^{\circ}$ flexed hip both in abduction and adduction, and adding internal and external rotation to pinch the torn labrum between the femoral head and acetabular rim (a useful video of how to do a hip examination for a labral tear is available at www.youtube.com/ watch? $=$ Rtp4oz0_3YY). Although painful or palpable clicking may indicate the presence of a labral tear, the physical exam shows low specificity. ${ }^{3}$

\section{What investigations are required if a labral tear is suspected?}

An MR arthrogram involving injection of contrast medium into the joint is typically used to identify labral tears ${ }^{5}$ (Figure 1). Because a labral tear dis-

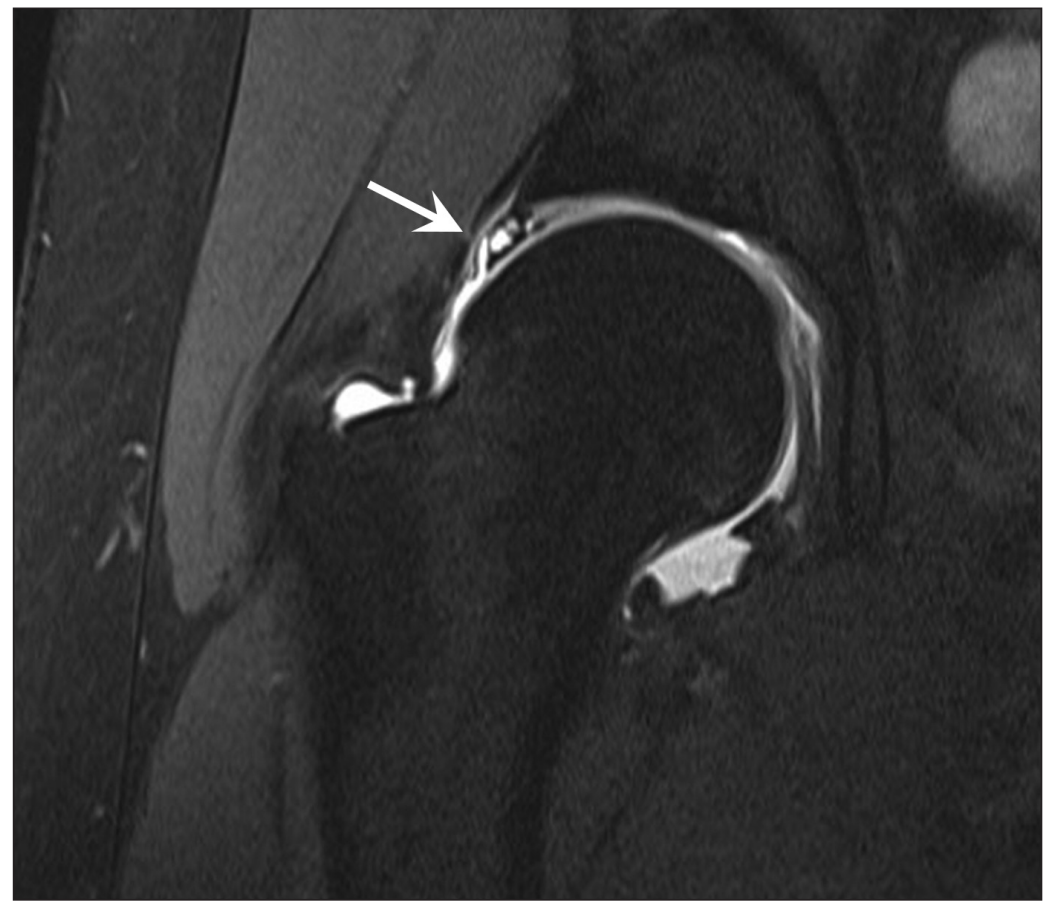

Figure 1: Magnetic resonance arthrogram of the right hip showing a tear of the superior labrum (arrow) with gadolinium contrast dissecting into the tear. 
rupts the seal of the synovial fluid within the joint, extravasation of the contrast medium shows the location and extent of the tear. For patients averse to intra-articular injection, high-resolution MR imaging may be a good substitute. ${ }^{6}$

\section{If imaging shows a labral tear, what are the treatment options for this patient?}

In this older patient, damage of the adjacent articular cartilage in her hip joint is likely, and labral débridement may not give definitive symptom relief.

Uncontrolled studies have shown that discrete sports-related labral tears may respond to arthroscopic hip surgery in younger athletic patients. ${ }^{7}$ However, a Canadian study involving 41 older patients ( $>45 \mathrm{yr}$; mean age $53.7 \mathrm{yr}$ ) showed poor results of surgery, with relatively high reoperation rates and minimal improvement in joint-specific and overall quality-of-life measures. ${ }^{8}$ The authors of a similar American study involving 30 patients (mean age $63.9 \mathrm{yr}$ ) also recommended caution in advising surgery in older patients because of poor two-year overall survival (70\%) and a reoperation rate of $37 \%$, most often requiring total hip arthroplasty. ${ }^{9}$

In this patient's age group, a labral tear may be a sign of a deteriorating joint rather than an isolated repairable lesion. Watchful waiting and non-load-bearing exercise may be a good option. Physiotherapy techniques currently include active and deep-tissue release in addition to progressive resisted stretching and strengthening activities. ${ }^{1}$ Poor response to conservative treatment and increasing disability may require revisiting surgery as a treatment option.

\section{Case revisited}

On further examination, rotational testing of the patient's flexed hip reproduced a painful click. The patient decided to proceed with an MR arthrogram, which showed a labral tear. Given the poor results of surgery in her age group, the patient decided not to consider surgery at this point and will seek conservative treatment with physiotherapy to maximize her overall flexibility and strength.

\section{References}

1. Groh MM, Herrera J. A comprehensive review of hip labral tears. Curr Rev Musculoskelet Med 2009;2:105-17.

2. McCarthy JC, Noble PC, Schuck MR, et al. The Otto E. Aufranc Award: The role of labral lesions to development of early degenerative hip disease. Clin Orthop Relat Res 2001;(393):25-37.

3. Reiman MP, Goode AP, Cook CE, et al. Diagnostic accuracy of clinical tests for the diagnosis of hip femoroacetabular impingement/labral tears: a systematic review with meta-analysis. Br J Sports Med 2014;49:811.

4. Lee AJ, Armour P, Thind D, et al. The prevalence if acetabular labral tears and associated pathology in a young asymptomatic population. Bone Joint J 2015;97-B:623-7.

5. Tian CY, Wang JQ, Zheng ZZ, et al. 3.0 T conventional hip MR and hip MR arthrography for the acetabular labral tears confirmed by arthroscopy. Eur J Radiol 2014;83:1822-7.

6. Sundberg TP, Toomayan GA, Major NM, et al. Evaluation of the acetabular labrum at 3.0-T MR imaging compared with 1.5-T MR arthrography: preliminary experience. Radiology 2006; 238:706-11.

7. Ayeni OR, Adamich J, Farrokhyar F, et al. Surgical management of labral tears during femoroacetabular impingement surgery: a systematic review. Knee Surg Sports Traumatol Arthrosc 2014; 22:756-62.

8. Wilkin G, March G, Beaule PE. Arthroscopic acetabular labral debridement in patients forty-five years of age or older has minimal benefit for pain and function. J Bone Joint Surg Am 2014;96:113-8.

9. Redmond JM, Gupta A, Cregar W, et al. Arthroscopic treatment of labral tears in patients aged 60 years or older. Arthroscopy 2015;31:1921-7.

Affiliations: Anishinaabe Bimaadiziwin Research Program (Kelly) and Department of Radiology (Panu), Sioux Lookout Meno Ya Win Health Centre, Sioux Lookout, Ont.; Department of Surgery (Gandhi), University of Toronto, Toronto, Ont.

Contributors: Len Kelly developed the concept and wrote the drafts of the article. Anukul Panu supplied the image and contributed to the content of the article. Rajiv Gandhi edited the drafts and contributed to the content. All of the authors approved the final version to be published and agreed to act as guarantors of the work.

Decisions is a series that focuses on practical evidence-based approaches to common presentations in primary care. The articles address key decisions that a clinician may encounter during initial assessment. The information presented can usually be covered in a typical primary care appointment. Articles should be no longer than 650 words, may include one box, figure or table and should begin with a very brief description $(75$ words or less) of the clinical situation. The decisions addressed should be presented in the form of questions. A box providing helpful resources for the patient or physician is encouraged. 